\title{
The Regional Vulnerability to Hypoglycemia-induced Neurotoxicity in Organotypic Hippocampal Culture: Protection by Early Tetrodotoxin or Delayed MK-801
}

\author{
Robert C. Tasker, ${ }^{a}$ Joseph T. Coyle, ${ }^{b}$ and James J. Vornov \\ Division of Pediatric Intensive Care, Departments of Anesthesiology and Critical Care Medicine, Neurology, Neuroscience, \\ and Psychiatry, The Johns Hopkins School of Medicine, Baltimore, Maryland 21205
}

Profound hypoglycemia selectively damages CA1 and the dentate gyrus of the hippocampus. We have examined the time course of hippocampal neuronal injury in organotypic cultures following in vitro "hypoglycemia," using the fluorescent vital dye propidium iodide to observe directly the regional distribution of early neuronal membrane injury in living cultures. The in vivo hippocampal pattern of hypoglycemic injury was reproduced by a $2 \mathrm{hr}$ exposure to glucosefree media, which resulted in simultaneous, selective propidium staining of $\mathrm{CA} 1$ and the dentate gyrus starting by 4 hr after exposure. After $24 \mathrm{hr}$ of recovery, CA3 remained spared. A similar pattern of propidium staining was produced by incubation of cultures for briefer periods in glucose-free medium containing $5 \mathrm{mM}$ 2-deoxyglucose (2-DG) to inhibit glycolysis. This "hypoglycemic" pattern and time course of neuronal injury was mimicked by $300 \mu \mathrm{M}$ aspartate but not by glutamate. The NMDA receptor antagonists MK-801 and CPP, but not the relatively selective non-NMDA receptor antagonist 6-cyano-7-dinitroquinoxaline-2,3-dione, prevented the development of propidium staining. MK- 801 protected against injury even if added to the recovery media $30 \mathrm{~min}$ after the insult, while TTX (10 $\mathrm{MM})$ protected only if added by the end of the exposure. The appearance of propidium staining after 4-6 hr of recovery was well correlated with histological observation of pyknotic neuronal nuclei in the injured regions. The characteristic hippocampal regional vulnerability of CA1 and the dentate gyrus to injury following profound hypoglycemia can be reproduced in organotypic hippocampal culture and appears to be mediated both by an early TTX-sensitive component and by a more prolonged period of toxic NMDA receptor activation, extending for at least $\mathbf{3 0} \mathrm{min}$ into the recovery period.

It has been suggested that NMDA receptor-mediated neurotoxicity is the primary mechanism of neuronal injury following profound hypoglycemia. Experimental evidence includes both the ultrastructural resemblance of hypoglycemic injury to ex-

\footnotetext{
Received Jan. 24, 1992; revised May 19, 1992; accepted June 2, 1992.

This research was supported by National Institutes of Health Grants NS 01310 to J.J.V. and NS 13584 to J.T.C.

Correspondence should be addressed to Dr. James J. Vornov, Department of Neurology, Johns Hopkins Hospital, Meyer 1-130, Baltimore, MD 21205.

Present address: Anaesthesia, Intensive Therapy and Respiratory Medicine Unit, Institute of Child Health, 30 Guilford Street, London, WCI, UK.

'Present address: Department of Psychiatry, Harvard Medical School, McLean Hospital, 115 Mill Street, Belmont, MA 02178.

Copyright (C) 1992 Society for Neuroscience $0270-6474 / 92 / 124298-11 \$ 05.00 / 0$
}

citatory amino acid neurotoxicity, a glial-sparing, axon-sparing, lesion of neurons that is most pronounced in the dendrites (Auer et al., 1985a,b), and the pharmacologic protection by NMDA receptor antagonists injected intracerebrally (Wieloch, 1985; Simon et al., 1986) or administered systemically (Westerberg et al., 1988; Papagapiou and Auer, 1990). In vitro pharmacologic studies with primary neuronal cultures have supported the hypothesis that glucose deprivation-induced neuronal injury is largely mediated by toxic activation of NMDA receptors (Monyer and Choi, 1988; Monyer et al., 1989; Facci et al., 1990). In vivo microdialysis analysis of the hippocampus during insulininduced hypoglycemia has demonstrated a 15-fold increase in aspartate and 3-fold increase in glutamate (Sandberg et al., 1986), suggesting that the toxic NMDA receptor activation may be mediated by an accumulation of endogenous excitatory amino acids in the extracellular space. This predominance of aspartate in hypoglycemia differs from the rise in extracellular glutamate observed following brief ischemia (Hagberg et al., 1985) and is probably due to a shift in the equilibrium of aspartate-aminotransferase toward aspartate during hypoglycemia, metabolizing tissue glutamate to aspartate (Agardh et al., 1978). Nevertheless, the relationships among these metabolic processes, endogenous neurotransmitter release, receptor-mediated neurotoxicity, and the regional vulnerability of the hippocampus remain poorly understood.

In a previous study, we utilized propidium iodide fluorescence to observe directly the time course of agonist-specific regional vulnerability to glutamate, NMDA, and kainate neurotoxicity in organotypic hippocampal culture (Vornov et al., 1991a), a preparation that largely preserves the intrinsic synaptic circuitry and regional differentiation of the hippocampus in long-term culture. Propidium iodide, a fluorescent dye that binds to nucleic acids, does not enter intact cells, but upon sufficient membrane injury, enters the cell and becomes concentrated in the nucleus, rendering it brightly fluorescent. By observing cultures in propidium containing media, the regional distribution of membrane injury can be detected with great sensitivity as it occurs in the living culture.

The regional vulnerability to glutamate toxicity could be clearly distinguished from that of NMDA. A 30 min exposure to $1 \mathrm{mM}$ glutamate caused membrane injury that first appeared in CA1 and then extended over hours to include CA3 and, finally, the dentate gyrus. In contrast, $10 \mu \mathrm{M}$ NMDA caused the simultaneous appearance of propidium staining in CA 1 and the dentate gyrus, sparing CA3 (Vornov et al., 1991a). In vivo, insulininduced hypoglycemia produces selective neuronal degeneration 
that resembles this NMDA pattern, affecting CA1 and the dentate gyrus, with sparing of the CA 3 pyramidal neurons (Auer et al., 1985a,b). If hypoglycemic injury in organotypic culture is mediated by direct, toxic activation of NMDA receptors, experimental glucose deprivation in organotypic hippocampal culture might reveal a similar distribution of early neuronal membrane injury related to NMDA receptors.

In the present article, we have investigated the hypothesis that hypoglycemic injury is caused by direct, toxic activation of NMDA receptors by endogenous aspartate. The time course and distribution of NMDA receptor-mediated injury following in vitro glucose deprivation, chemically induced "hypoglycemia" by 2 -deoxyglucose (2-DG) exposure, and aspartate exposure have been examined in organotypic hippocampal culture. Protection against injury by TTX and MK-801 were found to have distinct time courses, suggesting that sodium influx and/ or membrane depolarization precedes a more prolonged period of NMDA receptor-mediated neurotoxicity.

These results have been reported previously in abstract form (Tasker el al., 1991).

\section{Materials and Methods}

Organotypic hippocampal culture. Cultures were prepared as described previously using the method of Gähwiler (1981). Briefly, hippocampi of 4-7-d-old rat pups were rapidly removed under sterile conditions in a laminar flow hood and cut transversely into $425 \mu \mathrm{m}$ slices with a Mcllwain tissuc choppcr and a stcrilc razor blade. Each slice was transferred by pipette onto a drop of chicken plasma spread on a sterile glass coverslip; a drop of thrombin was subsequently added to the plasma to form a clot containing the slice. The coverslip bearing the slice was then transferred to a plastic culture tube containing $1.5 \mathrm{ml}$ of growth medium consisting of $25 \%$ heat-inactivated horse serum, $25 \%$ Earle's balanced salt solution (EBSS), and 50\% Eagle's basal medium (BME) supplemented with glutamine $(2 \mathrm{mM})$ and glucose $(6.5 \mathrm{gm} / \mathrm{liter})$. The sealed tubes were placed in a roller apparatus rotating at 10 revolutions $/ \mathrm{hr}$ in a dry incubator at $36^{\circ} \mathrm{C}$. The medium was changed thereafter once a week. Slices were maintained in culture for at least 2 weeks prior to use in these experiments, when they had flattened to near-monolayer thickness.

Solutions. The control medium for exposure experiments was an HEPES-buffercd salt solution (HBSS) containing (in mM) $143.4 \mathrm{NaCl}$, $5.4 \mathrm{KCl}, 1.2 \mathrm{MgSO}_{4}, 1.2 \mathrm{NaH}_{2} \mathrm{PO}_{4}, 2.0 \mathrm{CaCl}_{2}, 10 \mathrm{D}$-glucose, and 5.0 HEPES (free acid) titrated to $\mathrm{pH} 7.40$ with $\mathrm{NaOH}$. This medium is identical to that used in previous experiments with organotypic hippocampal cultures (Vornov et al., 199 la). The glucose-deficient medium consisted of HBSS without D-glucose. The solution used for rapid inhibition of glycolysis contained 2,5 , or $10 \mathrm{mM} \mathrm{2-DG}$ added to the glucose-deficient HBSS. The serum-free medium consisted of $75 \% \mathrm{BME}$ and $25 \%$ EBSS, supplemented with D-glucose $(6.5 \mathrm{gm} / \mathrm{liter})$ and glutamine ( $2 \mathrm{mM})$.

Propidium iodide fluorescence microscopy of neuronal injury in organotypic hippocampal cultures. The time course and distribution of hippocampal neuronal injury were observed directly using previously described techniques (Vornov et al., 1991a). Cultures were incubated with propidium iodide added to $2 \mathrm{ml}$ of the experimental solution (4.6 $\mu \mathrm{g} / \mathrm{ml}$ ) for a minimum of $15 \mathrm{~min}$ before being assessed with an inverted fluorescence microscope using a standard rhodamine filter set. Regional propidium staining was studied in a qualitative manner by noting the onset and distribution of fluorescence, and progression of intensity, if any, in each of the major hippocampal regions. Video images were captured with a high-sensitivity CCD camera (Videoscope CCD 200) and a video frame grabber card (Data Translation) in an Apple Macintosh IIcx computer with the program IMAGE (Wayne Rasband, National Institutes of Health, Bethesda, MD). Gain and pedestal settings for the CCD camera were held constant to standardize the sensitivity of the video images.

Histology of organotypic hippocampal cultures. At the end of experiments, cultures were fixed in methanol overnight at $4^{\circ} \mathrm{C}$, stained with thionin, and mounted on microscope slides. Blinded histologic evaluation of the thionin-stained cultures were made by one of the investi-

\section{Experimental Protocol}

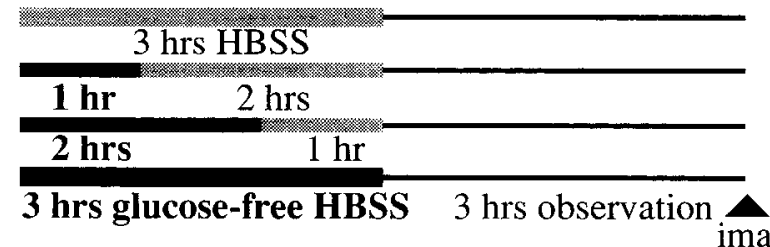

$\begin{array}{lcc}\text { 30 min } & 6 \text { hrs observation }\end{array}$

Roller tube recovery (18 hours)<smiles>C1=CC=C1</smiles>

Image propipidium staining, then methanol fix and thionin stain

Figure 1. Diagram of the experimental protocol. Experiments using glucose-free media to create hypoglycemia consisted of a $3 \mathrm{hr}$ exposure period in HBSS (thick bar) followed by a $3 \mathrm{hr}$ observation period in serum-free medium (thin bar). In experiments using 2-DG to create hypoglycemia, the HBSS exposure period was 30 min (thick bar) followed by a $6 \mathrm{hr}$ observation period in serum-free medium (thin bar). All experiments continued with roller tube recovery and then fixation in methanol and thionin staining. While multiple video micrographs were routinely obtained during the first $6 \mathrm{hr}$ of each experiment, an image was always obtained at the conclusion of the observation period (arrowheads) and at the end of the recovery period to document the time course and distribution of propidium staining.

gators (J.J.V). As described previously (Vornov et al., 1991a), each hippocampal region was graded using a four-point semiquantitative scale of the percentage of clearly pyknotic nuclei, where 0 was $<25 \%$ pyknotic, 1 was $25-50 \%, 2$ was $>50-75 \%$, and 3 was $>75-100 \%$.

Experimental design. The overall design of the experiments is illustrated in Figure 1. Under sterile conditions, cultures were removed from their tubes and placed in $35 \mathrm{~mm}$ plastic culture dishes containing $2 \mathrm{ml}$ of the serum-free medium with added propidium iodide. If significant propidium staining was observed in a culture during this screening step, it was not used in the experiment, as the staining was indicative of preexisting neuronal death. After screening, the medium was changed to HBSS (control, glucose-deficient, or 2-DG without glucose) and placed in a dry, room air incubator at $36^{\circ} \mathrm{C}$ for the period of exposure. Following experimental exposure, all cultures were washed twice with $2 \mathrm{ml}$ of fresh control HBSS, and then returned to serum-free medium containing propidium iodide and placed in an incubator with a moist $5 \% \mathrm{CO}_{2}$ atmosphere at $36^{\circ} \mathrm{C}$. After $6 \mathrm{hr}$ from the start of the experiment, cultures were returned to roller tubes containing $1.5 \mathrm{ml}$ of serum-free medium (without propidium iodide) for a further $18 \mathrm{hr}$. The fluorescence video micrographs in the figures were obtained either at the end of the $6 \mathrm{hr}$ observation period or at the conclusion of the experiment, at the end of the $18 \mathrm{hr}$ roller tube recovery. $\Lambda$ dditional controls for these cxperiments included cultures removed from their culture tubes without incubation in an experiment, as well as cultures undergoing the experimental protocols in the absence of added propidium iodide. Cultures were excluded from experimental analysis if (1) the culture failed the initial propidium screen before exposure, (2) the clot bearing the culture detached from its coverslip during the experiment, or (3) thionin staining of the culture after the experiment revealed any incomplete or absent hippocampal regions.

Some experiments were carried out solely in the roller tubes. Hippocampal cultures were exposed to $1.5 \mathrm{ml}$ of the glucose-deficient HBSS or control medium for $2 \mathrm{hr}$. This medium was then exchanged for 1.5 $\mathrm{ml}$ of the serum-free medium, which remained in the tube for a further $24 \mathrm{hr}$, before being changed to standard growth medium for another 6 $\mathrm{d}$ of recovery, when the experiment was ended.

Statistics. Nonparametric tests were used for the analysis of ordinal 

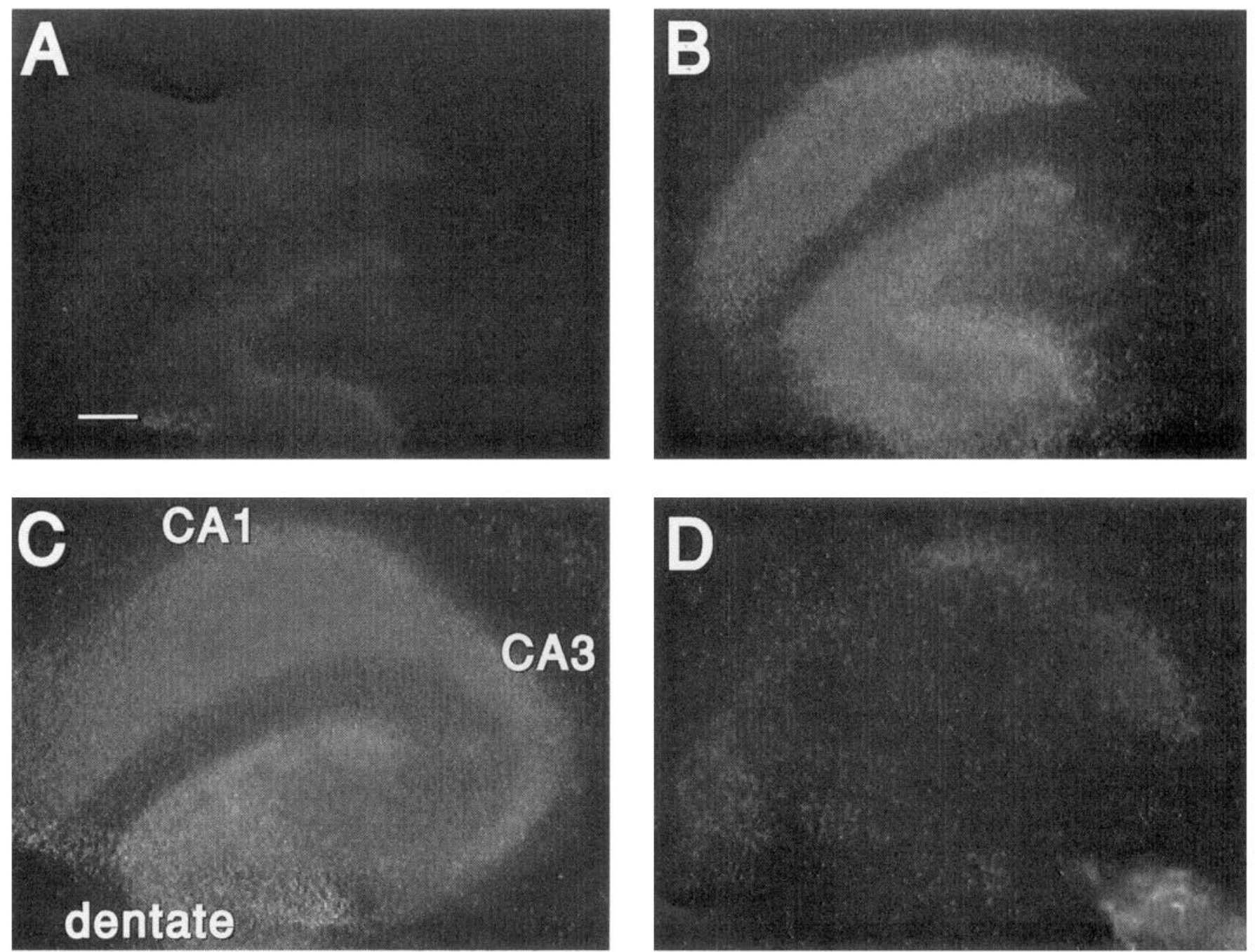

Figure 2. Propidium iodide fluorescence following incubation in glucose-free HBSS. $A$ and $B$, Two hour exposure: fluorescence video micrographs of propidium staining of an organotypic culture following a $2 \mathrm{hr}$ exposure to glucose-free HBSS. At the conclusion of the observation period, $4 \mathrm{hr}$ after the end of glucose deprivation, diffuse propidium staining is visible in CA1 and the dentate gyrus. $B$, At the end of the experiment following 18 more hours of recovery, propidium staining has become brighter and more punctate, but is still limited to CAl and the dentate gyrus. $C$, Three hour exposure. As illustrated by this culture, cultures exposed to glucose deprivation for $3 \mathrm{hr}$ demonstrated bright propidium staining in all regions at the conclusion of the experiment. $D$, Three hour exposure to HBSS. This culture illustrates the faint, diffuse propidium staining of CA1 and the dentate gyrus observed in control cultures incubated in HBSS for $3 \mathrm{hr}$ and allowed to recover. Scale bar $(A), 200 \mu \mathrm{m}$ for A-D.

data, Kendall's tau $B$ or tau $C$ for more than two groups, and the MannWhitney rank sum test for two groups.

\section{Results}

\section{Glucose deprivation}

In the initial series of experiments, the membrane injury produced in organotypic hippocampal cultures by increasing periods (control, 1, 2, and $3 \mathrm{hr}$ ) of glucose-deficient HBSS medium was assessed by fluorescence observation of propidium staining. In cultures exposed to the glucose-deficient medium for $2 \mathrm{hr}$, propidium staining was first observed after $4 \mathrm{hr}$ of recovery (three of three cultures). CAl and the dentate gyrus showed diffuse staining simultaneously, while CA3 exhibited no fluorescence (Fig. $2 A$ ). Over the rest of the $24 \mathrm{hr}$ of recovery, CA3 generally remained nonfluorescent and the staining of CAl and the dentate gyrus became more intense and well demarcated (Fig. 2B). When the duration of hypoglycemic exposure was increased to $3 \mathrm{hr}$, propidium staining began earlier, after only
$2 \mathrm{hr}$ of recovery, and was more florid, with significant staining in all hippocampal regions (four of four cultures) (Fig. 2C). These patterns were not observed in cultures exposed to control HBSS for $3 \mathrm{hr}$ (three of three cultures) or in those exposed to a glucose-deficient medium for $1 \mathrm{hr}$ (three of three cultures).

While the fluorescence observations indicated regionally specific patterns of membrane injury following glucose deprivation, cultures did not tolerate prolonged HBSS incubation well. In both the controls and the $1 \mathrm{hr}$ hypoglycemic cultures, minimal fluorescence was present in parts of CAl and the dentate hilus after $24 \mathrm{hr}$ of recovery (Fig. 2D). In addition, the plasma clot containing the organotypic hippocampal culture tended to detach from the glass coverslip more frequently following prolonged HBSS exposure, making histologic examination of cultures after recovery of more than $24 \mathrm{hr}$ difficult. The injury of control cultures was due to the lack of amino acids and vitamins in the HBSS. It was not due to the removal of serum, as cultures tolerate several days of incubation in the serum-free medium. 

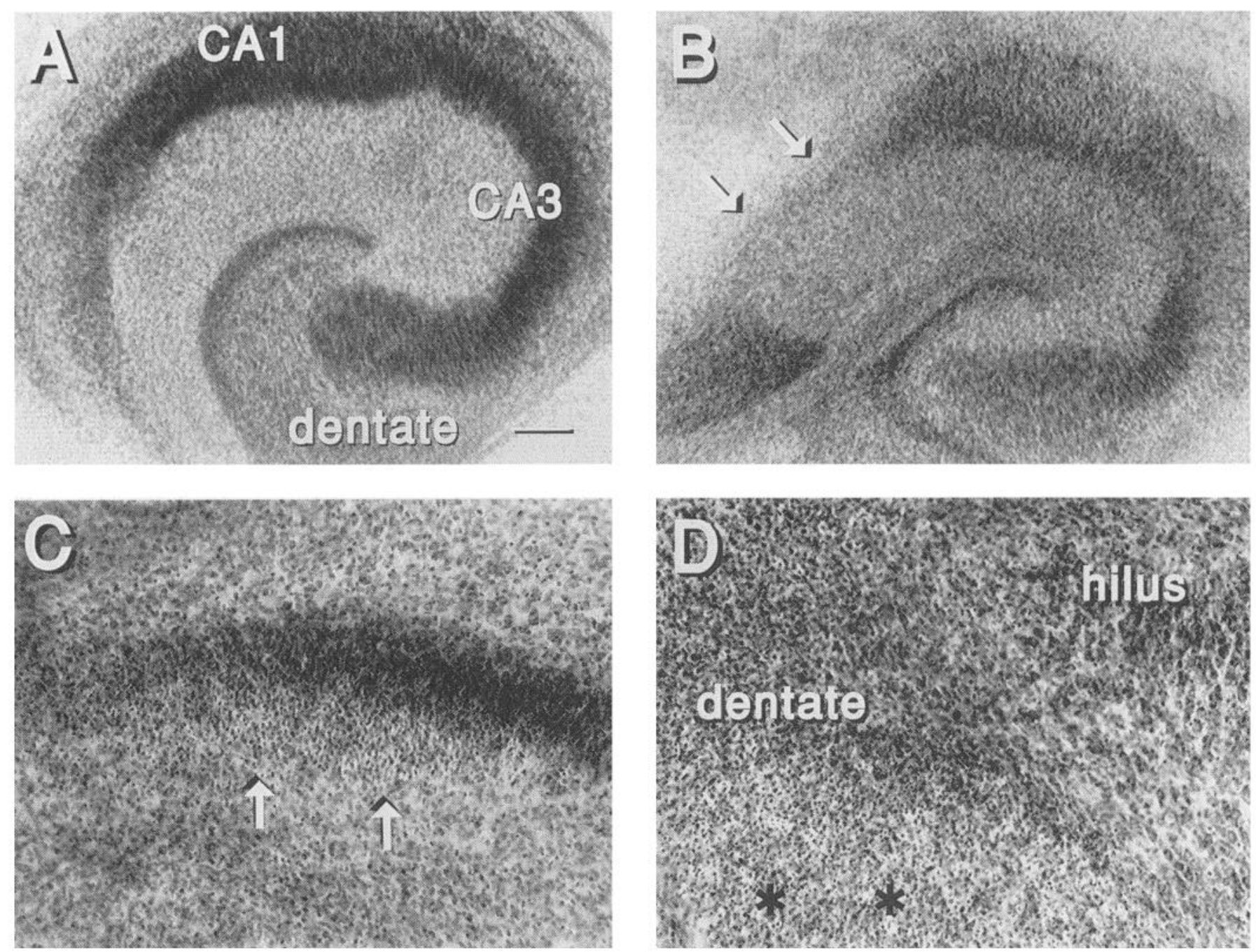

Figure 3. Histologic changes following exposure to glucose-free HBSS: video micrograph of typical cultures stained with thionin after a 2 hr exposure in roller tubes to HBSS with or without glucose followed by $7 \mathrm{~d}$ of recovery. $A$, Control culture. Preservation of organotypic organization is demonstrated. $B$, Glucose-free HBSS, 2 hr. An area of neuronal pyknosis can be plainly seen in CA1 near the subiculum (arrows). Pyknosis also was observed in the dentate gyrus. $C$ and $D$, Glucose-free HBSS, $2 \mathrm{hr}$ : higher-power view of a less severely affected culture, demonstrating a patch of neuronal pyknosis in CA1 (arrows in $C$ ) and the inferior blade of the dentate gyrus (asterisks in $D$ ). Scale bar $(A): 200 \mu \mathrm{m}$ for $A$ and $B ; 80 \mu \mathrm{m}$ for $C$ and $D$.

If the protocol was changed so that the exposure to either glucose-deficient medium or control-HBSS was carried out in the roller tubes, without transfer to dishes and fluorescence observation, the recovery period could be extended to $7 \mathrm{~d}(1 \mathrm{~d}$ in serum-free medium followed by $6 \mathrm{~d}$ in standard growth medium). As shown in Figure 3, neuronal pyknosis was seen in the CA1 region and the dentate gyrus, but not in CA3, correlating with the distribution of propidium staining observed in the earlier experiments. Within CA1 some cultures exhibited injury throughout, while others had a characteristic "bite" in CA1 nearest the subiculum. Pyknosis in the dentate was observed at the "crest" and inferior blade. Pyknosis was always detectable, but was rarely greater than $25 \%$ of all neurons in the affected hippocampal regions. These patterns of pyknosis were not present in control cultures.

\section{Metabolic inhibition with 2-DG}

To study further the early time course of in vitro hypoglycemia with a more robust injury, we used 2-DG to block glycolysis, which allowed us to shorten the period of experimental exposure from $2 \mathrm{hr}$ to $30 \mathrm{~min}$, eliminating any injury of control cultures and reliably producing histologic changes after $24 \mathrm{hr}$ of recovery. Cultures exposed to a glucose-deficient medium supplemented with $5 \mathrm{mM} 2$-DG for $30 \mathrm{~min}$ demonstrated the same patterns and progression of propidium iodide staining observed in the initial series of experiments following $2 \mathrm{hr}$ of hypoglycemic exposure (Fig. 4). The earliest propidium staining occurred simultaneously in CA1 and the dentate gyrus between 2 and $4 \mathrm{hr}$ of recovery and progressed over the $24 \mathrm{hr}$ of recovery. Some cultures also exhibited staining developing in CA3, though its intensity and progression lagged behind earlier changes in CA1 and the dentate gyrus in all instances. These effects were not prevented by further supplementing the $5 \mathrm{mM}$ 2-DG hypoglycemic medium with fructose-1-6-biphosphate, pyruvate, or glutamine, during and after the $30 \mathrm{~min}$ exposure. Cultures exposed to $5 \mathrm{mM} 2-\mathrm{DG}$ in HBSS containing $30 \mathrm{mM}$ glucose remained intact. Similarly, as described previously, control cultures exposed to the normoglycemic HBSS medium for $30 \mathrm{~min}$ remained nonfluorescent over the $24 \mathrm{hr}$ of the experiment.

The effects of exposure to increasing concentrations of 2-DG 

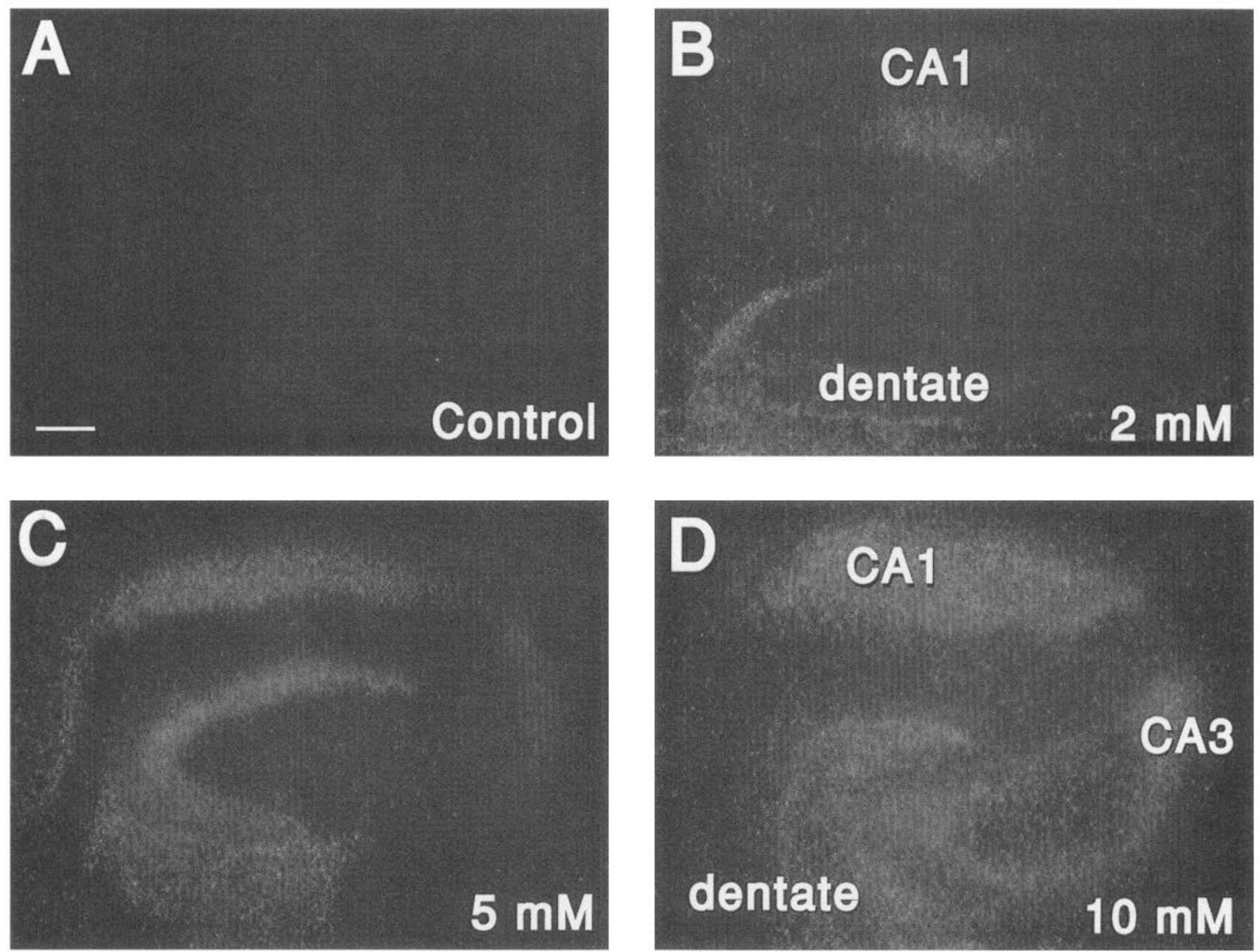

Figure 4. Propidium iodide fluorescence after exposure to increasing concentrations of 2-DG: fluorescence video micrographs of propidium staining in organotypic cultures following $30 \mathrm{~min}$ exposures to HBSS and 2-DG at the concentrations indicated in the lower right corner of each panel. All images were obtained at the end of the experiment. $A$, Control cultures did not develop additional fluorescence during the experiment. $B$, Limited staining, confined to CA1 and the dentate gyrus following $2 \mathrm{mM}$ 2-DG. $C$, Following $5 \mathrm{mM}$ 2-DG, propidium staining in CA1 and the dentate gyrus is greater, with less propidium staining in CA3. D, 2-DG at $10 \mathrm{mM}$ generally caused staining throughout the culture. The numbers of cultures displaying each of these patterns are summarized in Table 1 . Scale bar $(A), 200 \mu \mathrm{m}$ for $A-D$.

Table 1. Patterns of propidium staining at 6 and $24 \mathrm{hr}$ after exposure to 2-DG

\begin{tabular}{lllll} 
Condition & $\begin{array}{l}\text { No } \\
\text { staining }\end{array}$ & $\begin{array}{l}\text { CA1/ } \\
\text { dentate }\end{array}$ & $\begin{array}{l}\text { CA1/ } \\
\text { dentate } \\
>\text { CA3 }\end{array}$ & All \\
\hline $\begin{array}{l}\text { Six hour recovery } \\
\text { Control }\end{array}$ & 4 & - & - & - \\
$2 \mathrm{~mm}$ 2-DG & 2 & 3 & - & - \\
$5 \mathrm{~mm}$ 2-DG & - & 7 & 2 & - \\
$10 \mathrm{~mm}$ 2-DG & - & 1 & 6 & - \\
Twenty-four hour recovery & & & \\
Control & 4 & - & - & - \\
$2 \mathrm{~mm}$ 2-DG & 1 & 4 & - & - \\
$5 \mathrm{~mm}$ 2-DG & - & - & 6 & 3 \\
$10 \mathrm{~mm}$ 2-DG & - & - & 2 & 5
\end{tabular}

The pattern of propidium staining was assessed 6 and $24 \mathrm{hr}$ after a 30 min exposure to 2-DG in glucose-free HBSS at the concentrations indicated. The numbers of cultures displaying each pattern of staining are shown in the table. There was a significant correlation between increasing 2-DG concentration and more extensive propidium staining at each observation time ( $p<0.001$, Kendall's tau B). (control, $2 \mathrm{mM}, 5 \mathrm{mM}$, and $10 \mathrm{mM}$ ) are illustrated in Figure 4. The concentration dependence of 2-DG-induced membrane injury is summarized in Table 1, with respect to the four patterns observed in the initial series of experiments (none, CAl and dentate gyrus, predominantly $\mathrm{CAl}$ and dentate gyrus with diffuse staining in $\mathrm{CA} 3$, and uniform staining in all hippocampal regions). Histologic evidence of injury after $24 \mathrm{hr}$ of recovery correlated well with the progression of early propidium staining, but varied in severity depending upon the concentration of 2-DG. As observed previously, nuclei of affected neurons were severely pyknotic and cytoplasmic Nissl staining was lost, so that the neurons appeared as small, dark, pyknotic nuclei surrounded by an area devoid of Nissl staining. The most severe histologic changes were seen following exposure to $10 \mathrm{mM}$ 2-DG, where there was widespread pyknosis in all hippocampal regions. At lower concentration ( $2 \mathrm{mM}$ and $5 \mathrm{mM}$ ), degeneration was limited to the neuronal population of CAl and the dentate gyrus (Fig. 5A); glia appeared to be unaffected. Following exposure to $5 \mathrm{mM}$ 2-DG, there was a sharp demarkation between the nor- 

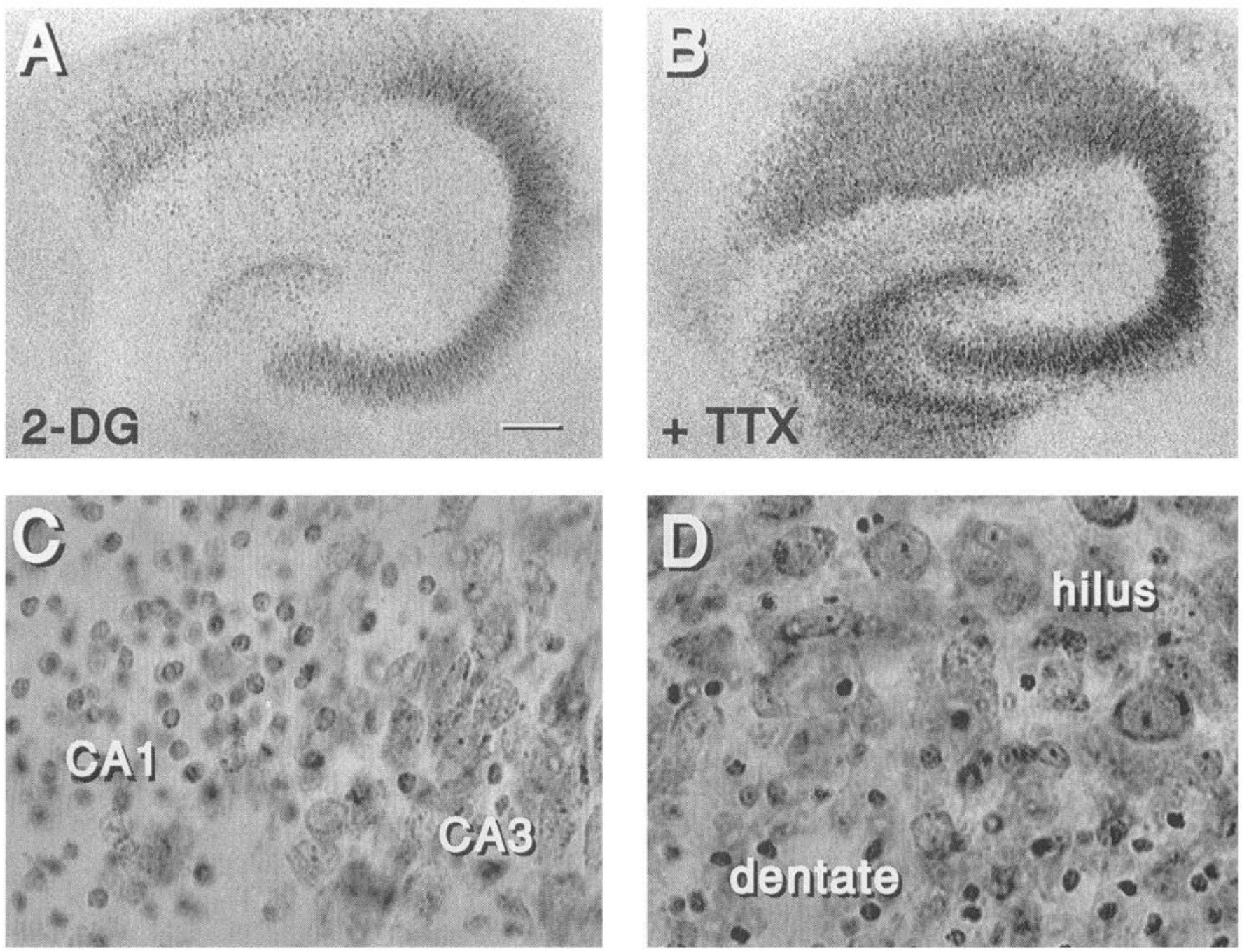

Figure 5. Histologic changes following exposure to 2-DG: video micrographs of typical cultures stained with thionin after a 30 min exposure to 2-DG followed by $24 \mathrm{hr}$ of recovery. $A, 2-\mathrm{DG}$ at $5 \mathrm{mM}$. Neuronal loss in CAl and the dentate gyrus is evident. $B, 2-\mathrm{DG}$ at $5 \mathrm{mM}$, with TTX added immediately at the beginning of recovery period. The culture is indistinguishable from control. $C$ and $D, 2$-DG at $5 \mathrm{mM}$ : higher-power views demonstrating sharp demarcation of regional vulnerability. In $C$, the pyknotic neuronal nuclei of CAl border on the normal appearing neurons CA3, while in $D$, pyknotic nuclei of the dentate granule cells are juxtaposed with the large neurons of the hilus. Occasional small granule cells are preserved. Scale bar $(A): 200 \mu \mathrm{m}$ for $A$ and $B ; 40 \mu \mathrm{m}$ for $C$ and $D$.

mal-appearing neurons of $\mathrm{CA} 3$ and the pyknotic nuclei of CA1 (Fig. 5C).

Degeneration of the dentate granule cells was in striking contrast to the preservation of the large hilar neurons (Fig. 5D). Thus, the selective injury of CA1 and the dentate gyrus with CA3 sparing following exposure to $5 \mathrm{mM} 2-\mathrm{DG}$, seen with propidium staining starting after $2-4-\mathrm{hr}$ of recovery and developing over the entire $24 \mathrm{hr}$ of recovery, was confirmed histologically. Much less severe changes were observed in cultures exposed to $2 \mathrm{mM} 2-\mathrm{DG}$, with scattered pyknotic nuclei localized to part of CA1. The histologic grading of cultures summarized in Figure 6 illustrates the concentration dependence of histologic changes, as well as the differences in regional vulnerability.

\section{Comparison of hypoglycemic, NMDA, aspartate, and glutamate neurotoxicity}

The selective injury of CAl and the dentate gyrus observed following in vitro hypoglycemia bore striking similarities to the pattern of injury caused by exposure to NMDA (Vornov et al., 1991a). We have previously reported that a $30 \mathrm{~min}$ exposure to $100 \mu \mathrm{M}$ NMDA causes propidium staining of neurons throughout the culture, beginning during the exposure and increasing to a maximum over the first $6 \mathrm{hr}$ of recovery. When the concentration of NMDA is reduced to $10 \mu \mathrm{M}$, selective propidium staining is seen in CAl and the dentate gyrus, beginning after $2-4 \mathrm{hr}$ of recovery and increasing during the entire $24 \mathrm{hr}$ recovery period. CA3 was spared.

Aspartate, at a concentration of $300 \mu \mathrm{M}$, caused selective staining in CA1 and the dentate gyrus, with a slower time course than NMDA (Fig. 7). In contrast to the similar patterns of injury induced by hypoglycemia, NMDA, and aspartate, glutamate produced the same distinct, progressive pattern described previously (Vornov et al., 1991a), which begins in CA1, extends to $\mathrm{CA} 3$, and finally includes the dentate gyrus. After $6 \mathrm{hr}$ of recovery following exposure to $1 \mathrm{mM}$ glutamate, staining was predominantly in CA1 and CA3 (Fig. 7). 


\section{Control}

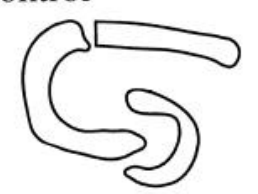

2-DG

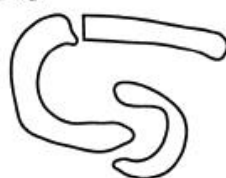

$2 \mathrm{mM}$
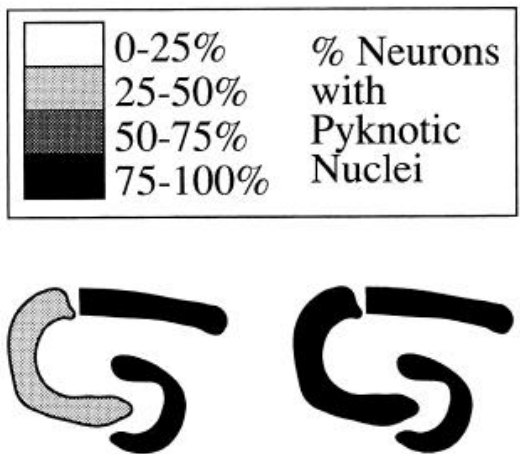

$5 \mathrm{mM}$

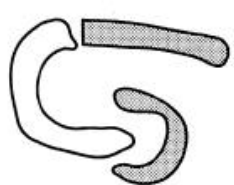

$300 \mu \mathrm{M}$

$100 \mu \mathrm{M}$

2-DG $5 \mathrm{mM}$

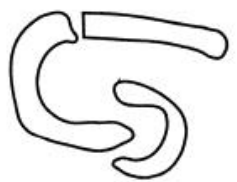

+ TTX

immediate

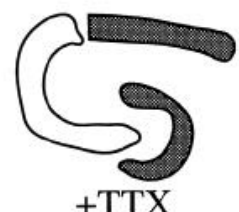

$5 \mathrm{~min}$

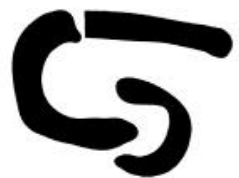

$10 \mathrm{mM}$

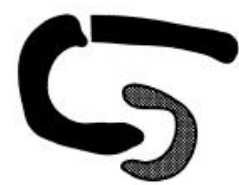

$1 \mathrm{mM}$

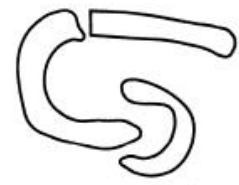

$+\mathrm{MK}-801$

$30 \mathrm{~min}$
Figure 6. Summary of histologic observations. As described in Materials and Methods, neuronal nuclear pyknosis in Nissl-stained cultures was graded on the four-point scale shown in the figure. The median score for each region under each condition is represented by the gray scale. Exposures to 2-DG and aspartate were $30 \mathrm{~min}$ at the concentrations shown. The bottom row represents cultures exposed to $5 \mathrm{mM} 2$ DG followed by TTX $(10 \mu \mathrm{M})$ or MK-801 $(30 \mu \mathrm{M})$ after exposure, with the delays shown: immediately following $2-\mathrm{DG}, 5$ or $30 \mathrm{~min}$ delayed. Each diagram represents the median score for four or more cultures.

\section{Protection by $N M D A$ receptor antagonists}

The effects of competitive receptor antagonists on in vitro "hypoglycemic" neurotoxicity were evaluated by propidium iodide fluorescence and histology in cultures exposed to $5 \mathrm{mM} 2-\mathrm{DG}$ in hypoglycemic media (Fig. 8). CPP (3-(2-carboxypiperazin-4yl) propyl-1-phosphonic acid), a competitive antagonist of the NMDA receptor, when present at $100 \mu \mathrm{M}$ during and after the exposure prevented development of propidium staining, as did MK-801, a noncompetitive NMDA receptor antagonist. Histology at $24 \mathrm{hr}$ confirmed this protection, with no significant difference compared with controls. 6,7-Cyano-7-dinitroquinoxaline-2,3-dione (CNQX), a relatively selective, competitive antagonist of non-NMDA receptors, failed to delay or prevent propidium iodide staining and neuronal pyknosis following 2-DG exposure.

MK-801 even protected against the $5 \mathrm{mM}$ 2-DG injury when added $30 \mathrm{~min}$ into the recovery period (Fig. 8, Table 2), but not if added after $1 \mathrm{hr}$ of recovery. When added $30 \mathrm{~min}$ after the end of the 2-DG exposure, $30 \mu \mathrm{M}$ was the lowest concentration of MK-801 that would completely prevent propidium staining and histologic injury. Cultures exposed to $10 \mu \mathrm{M}$ MK- 801 from $30 \mathrm{~min}$ of recovery were also not different from controls histologically, indicating that the minor propidium staining observed in CAl and the dentate gyrus (two of four cultures) or the dentate gyrus alone (two of four cultures) was not associated with a detectable pyknosis after $24 \mathrm{hr}$ of recovery.

The finding that MK-801 could be used to limit toxicity in the recovery period after in vitro hypoglycemia suggests that a period of toxic NMDA receptor activation follows the end of the exposure period. We wondered whether this toxic receptor activation was dependent on neuronal activity causing release of an endogenous agonist, possibly aspartate. If so, the sodium channel blocker TTX should attenuate hypoglycemic injury by preventing axonal impulse transmission and thus prevent synaptic release of endogenous agonist. TTX attenuated propidium iodide staining if present immediately after exposure to 2-DG, beginning at the time of washing the cultures (five of five cul-
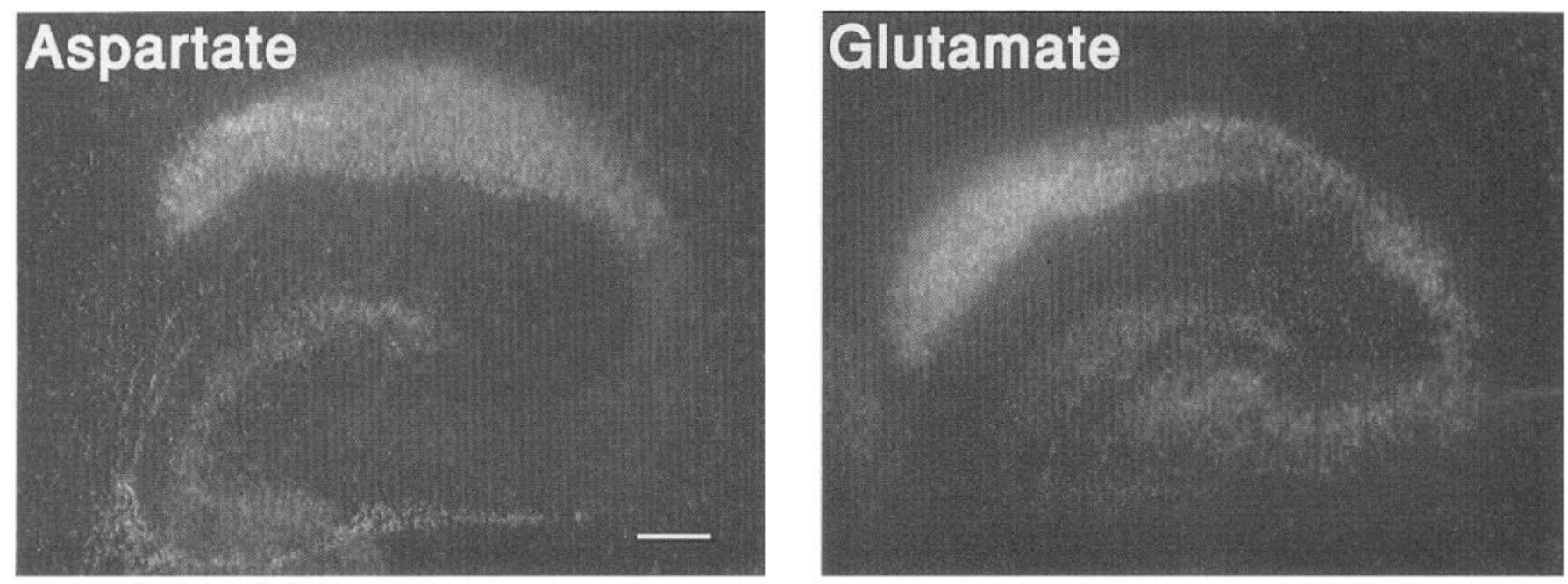

Figure 7. Propidium iodide fluorescence following exposure to aspartate $(300 \mu \mathrm{M})$ and glutamate: fluorescence video micrographs of propidium staining obtained at the end of the experiment following $30 \mathrm{~min}$ exposures to aspartate or glutamate. Aspartate caused propidium staining that was most intense in CA1 and the dentate gyrus, while glutamate caused staining that was greatest in CA1 and least in the dentate. Aspartate exposure more closely reproduced the pattern of staining caused by hypoglycemic conditions. Scale bar $(A), 200 \mu \mathrm{m}$ for $A$ and $B$. 

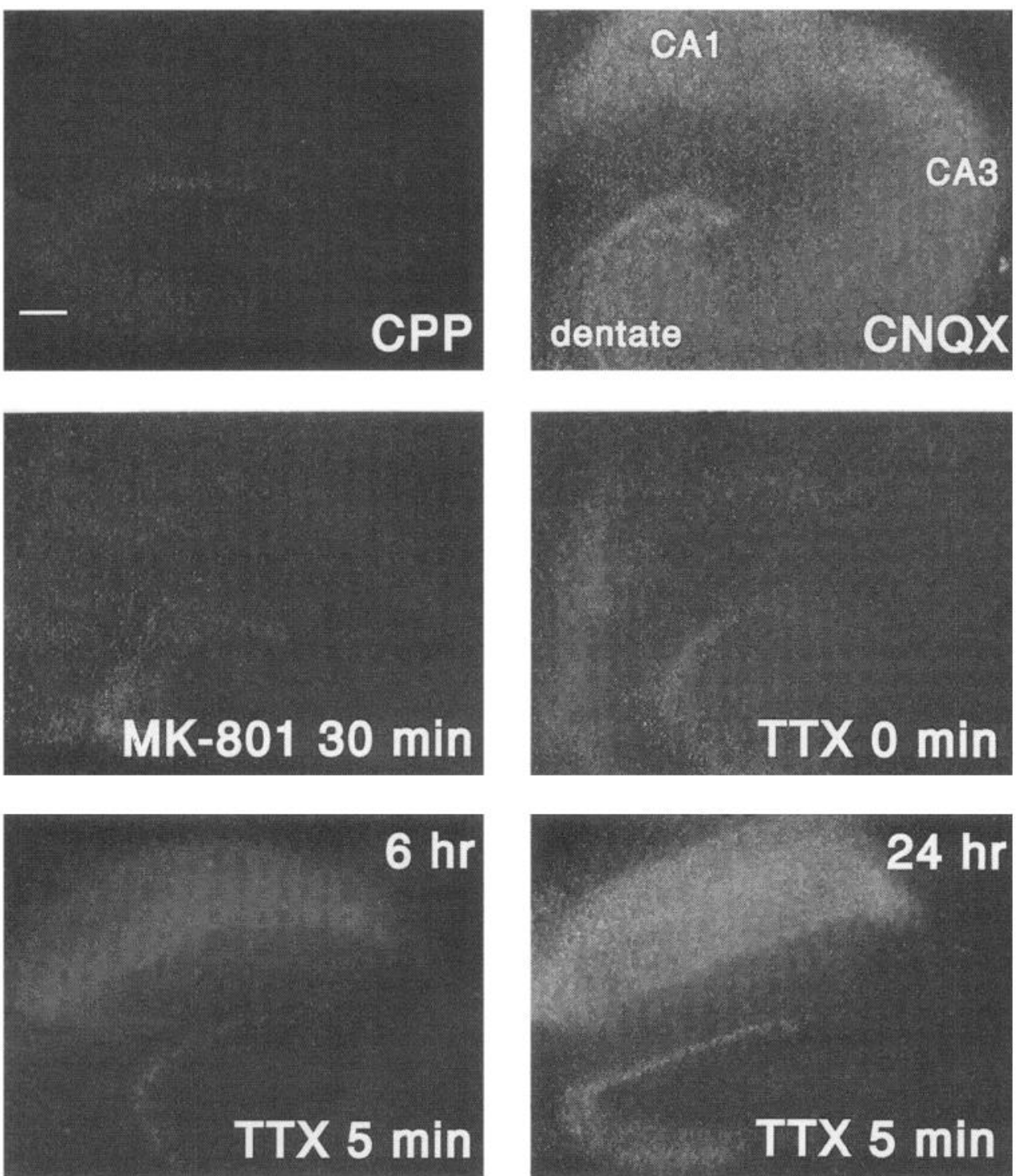

Figure 8 . Protection by NMDA receptor antagonists and TTX: fluorescence video micrographs of propidium staining obtained at the end of the experiment following $30 \mathrm{~min}$ exposures to 2-DG. Cultures were protected by $\mathrm{CPP}(100 \mu \mathrm{M})$ but not CNQX $(100 \mu \mathrm{M})$ when these compounds were present during and after 2-DG exposure (top row). MK-801 $(30 \mu \mathrm{M})$ protected even if added $30 \mathrm{~min}$ after the end of 2-DG exposure, while TTX $(10 \mu \mathrm{M})$ fully protected only if added immediately after 2-DG exposure (middle row). If the addition of TTX was delayed by $5 \mathrm{~min}$ (bottom row), propidium staining may have been modified but was not prevented. Scale bar (top left), $200 \mu \mathrm{m}$ for all micrographs. tures). Histologic preservation was well correlated with prevention of propidium staining (Figs. $5 B, 6$ ). However, as shown in Figure 8 , when TTX was added to the recovery media $5 \mathrm{~min}$ after the exposure to 2-DG, propidium staining was not prevented. The delayed TTX addition still altered the appearance of the propidium staining. Instead of the usual clearly defined fluorescent nuclei, there was an uncharacteristic, diffuse "haze" in the affected areas, suggesting an alteration in the development of neuronal injury. Indeed, these cultures had less consistent histologic changes, with only four of six cultures demonstrating significant pyknosis in CAl (Fig. 6).

\section{Discussion}

These experiments strongly support the hypothesis that the neuronal damage that occurs in the hippocampus following profound hypoglycemia is mediated, at least in part, by direct toxic activation of NMDA receptors by an endogenous agonist, aspartate. We have made three principal observations. (1) The characteristic in vivo regional vulnerability of the hippocampus to hypoglycemia, selective injury of $\mathrm{CAl}$ and the dentate gyrus, with sparing of $\mathrm{CA} 3$, can be reproduced in vitro, in organotypic
Table 2. Prevention of propidium staining at 6 and $24 \mathrm{hr}$ by MK-801 added $30 \mathrm{~min}$ after exposure to 2-DG

\begin{tabular}{ccccc} 
[MK-801] & $\begin{array}{l}\text { No } \\
\text { staining }\end{array}$ & $\begin{array}{l}\text { Dentate } \\
\text { only }\end{array}$ & $\begin{array}{l}\text { CA1/ } \\
\text { dentate }\end{array}$ & All \\
\hline $\begin{array}{c}\text { Six hour recovery } \\
0\end{array}$ & - & - & 9 & - \\
$1 \mu \mathrm{M}$ & - & - & 3 & - \\
$10 \mu \mathrm{M}$ & 1 & 2 & 1 & - \\
$30 \mu \mathrm{M}$ & 3 & 1 & - & - \\
$100 \mu \mathrm{M}$ & 3 & 1 & 1 & - \\
Twenty-four hour recovery & - & - & 6 & 3 \\
0 & - & - & 2 & 1 \\
$1 \mu \mathrm{M}$ & - & 2 & 2 & - \\
$10 \mu \mathrm{M}$ & - & 4 & - & - \\
$30 \mu \mathrm{M}$ & 1 & 3 & 1 & - \\
$100 \mu \mathrm{M}$ & & & & \\
\hline
\end{tabular}

The pattern of propidium staining was assessed 6 and $24 \mathrm{hr}$ after a $30 \mathrm{~min}$ exposure to $5 \mathrm{~mm}$ 2-DG in glucose-free HBSS. MK- 801 was added after $30 \mathrm{~min}$ of recovery at the concentrations indicated. The numbers of cultures displaying each pattern of staining are shown in the table. There was a significant correlation between increasing MK-801 concentration and less extensive propidium staining at each observation time ( $p<0.001$, Kendall's tau B). 
hippocampal cultures, either by exposure to glucose-deficient medium, or by metabolic inhibition of glycolysis. (2) This toxicity can be mimicked by brief exposure to NMDA, or the endogenous excitatory amino acid agonist L-aspartate, but not L-glutamate. It can be prevented by the NMDA receptor antagonists MK-801 or CPP, but not by the relatively selective non-NMDA receptor antagonist CNQX. Taken together, these findings suggest that hypoglycemia-induced neuronal injury is mediated predominantly by activation of the NMDA type of excitatory amino acid receptor, with L-aspartate as a likely endogenous agonist. (3) MK-801 protects even if administration is delayed for $30 \mathrm{~min}$ into the recovery period, but TTX must be immediately present in the glucose-containing wash to provide substantial protection from membrane injury, suggesting that the hypoglycemia-induced toxic NMDA receptor activation extends into the recovery period, significantly beyond an earlier period of sodium influx and/or depolarization, which is a preceding step in toxic NMDA receptor activation in hypoglycemia-induced neurotoxicity.

In the present series of experiments, either glucose deprivation or blockade of glycolysis with 2-DG resulted in a reproducible pattern of neuronal injury demonstrated by propidium staining, which was limited to CAl and the dentate gyrus. Like other vital dye exclusion methods, propidium iodide fluorescence is indicative of significant membrane injury (Jones and Senft, 1985; Lemasters et al., 1987; Favaron et al., 1988; Macklis and Madison, 1990) and has been previously correlated with lactate dehydrogenase release and degree of histologic changes in glutamate neurotoxicity in organotypic hippocampal cultures (Vornov et al., 1991a). While propidium staining is often cited as evidence of loss of cellular viability, it is not actually a direct measure of irreversible cellular necrosis. In this report, as in our previous experiments using propidium fluorescence to detect injury, we have exploited propidium to delineate the time course and distribution of injury, relying on histologic changes for confirmation. In the present experiments, we have again confirmed that bright, punctate propidium staining is well correlated with the later histologic distribution of neuronal pyknosis. Under some conditions, we have observed a dimmer, more diffuse pattern of propidium staining, which was not as well correlated with widespread pyknosis after $24 \mathrm{hr}$. These patterns are qualitatively easily distinguished, but raise the possibility that, under some conditions, membrane injury sufficient to allow propidium entry does not invariably lead to cell death. Given the value of propidium staining for sensitively detecting membrane injury, the morphologic correlates of propidium staining are deserving of further investigation, especially at the electron $\mathrm{mi}-$ croscopic level. Ultrastructural evidence of small membrane discontinuities in dendrites has been reported following ischemia in the rat (Diemer and Ekstrom von Lubitz, 1983; Johansen et al., 1984). In hypoglycemia in vivo, ultrastructural dendritic injury occurs more rapidly than in ischemia (Auer et al., 1985b). In vivo, the rapidity of ultrastructural injury is correlated with the severity of the insult (Auer et al., 1985b). Similarly, we have found that more severe injury is associated with more rapid onset of propidium staining. At present, we are unable to correlate the onset of propidium staining with previous ultrastructural studies of hypoglycemia in vivo.

Creation of "hypoglycemic" injury by simple glucose deprivation presented a number of technical problems, which led to the use of $2-\mathrm{DG}$ to produce a more rapid, reliable injury. The regional distribution of injury was similar for both models of injury. It required hours of simple glucose deprivation to cause injury, presumably because glycogen stores in cultures can be quite substantial. Variability can be introduced by differences in glycogen content between culture regions and between cultures. The insult caused by simple glucose deprivation may thus be slow in onset and unevenly distributed throughout the culture. The use of 2-DG alleviates the problems of prolonged incubation in the exposure medium and probably produces a block in glycolysis that is rapid and uniform in onset. Nevertheless, some uncertainty exists regarding the end of the period of glucose deprivation. Since 2-DG is transported into cells and phosphorylated, large amounts of phosphorylated 2-DG could accumulate in cells during exposure and glycolysis might resume slowly. In experiments in non-neuronal cells, nontoxic effects of high concentrations of 2-DG can be reversed within minutes of removal (Barry et al., 1987). While the duration of block of glycolysis was not measured in these experiments, two observations imply that it was limited in duration. First, the effects of $5 \mathrm{mM} 2-\mathrm{DG}$ could be blocked by $30 \mathrm{mM}$ glucose, the concentration of glucose present in the recovery medium. When present simultaneously, glucose will also compete for uptake of 2-DG into cells, so that the effects of glucose in the recovery medium will be less, but the restored glucose should be just as avidly transported and rapidly overcome any intracellular block of glycolysis. Second, TTX completely protected cultures when added immediately upon removal of 2-DG, but failed to protect 5 min later, implying that within $5 \mathrm{~min}$ of $2-\mathrm{DG}$ removal, the metabolic state of the culture had been critically altered. As discussed below, further experiments investigating the metabolic recovery of cultures are critical to understronding the delayed protective effects of MK-801.

Whether or not 2-DG was present, glucose deprivation caused injury of organotypic cultures in a pattern that was similar to that described in adult animals. In animal studies of profound hypoglycemia, enough to induce $30 \mathrm{~min}$ of isoelectricity, neurotoxicity in the hippocampus is limited to dense necrosis at the crest of the dentate gyrus and a gradient of increasing selective neuronal necrosis medially in CAl (i.e., a lessening gradient of neuronal necrosis toward the border with CA3) (Auer et al., 1985a,b; Auer and Siesjo, 1988). It has been proposed that this selective distribution of damage reflects extracellular or cerebrospinal fluid-borne excitatory amino acid neurotoxicity, which affects the brain regions and neurons in closest proximity to the lateral ventricle (Auer et al., 1984). The relative resistance of $\mathrm{CA} 3$ has been similarly explained, with damage occurring in this portion of the hippocampal neuronal cell band last, after CA1 and the dentate gyrus, when hydrocephalus is present and a portion of CA 3 abuts the enlarged ventricle. The protective effects of NMDA antagonists in animal models imply that the endogenous toxin is an excitatory amino acid neurotransmitter; microdialysis experiments describing a 15-fold increase in extracellular aspartate over baseline, with smaller increases in glutamate, suggest that aspartate is the endogenous, toxic agonist.

The consistent pattern of injury produced by NMDA, aspartate, glucose deprivation, and blockade of glycolysis by 2-DG described here supports the simple hypothesis that regional sensitivity of the hippocampus to direct NMDA receptor activation accounts for the sensitivity to hypoglycemia. Since the most vulnerable regions in the hippocampus are those with the highest density of NMDA receptors in vivo (Monaghan et al., 1983; Greenamyre et al., 1985), the simplest hypothesis is that recep- 
tor density is the primary determinant of vulnerability. Our observations did differ somewhat from previous in vivo results. Generally, we observed an almost uniform injury within the vulnerable regions, without any apparent intraregional gradient or predilection. However, with a threshold in vitro insult, $2 \mathrm{hr}$ of glucose deprivation in the roller tubes, there appeared to be a gradient in toxicity observed in CA1, which was greatest near CA3, the opposite from the in vivo pattern. Pyknosis was also more pronounced at the crest and in the inferior blade of the dentate gyrus, concordant with the in vivo pattern.

Proximity to the ventricle cannot be invoked in these experiments, since, of course, organotypic cultures are uniformly exposed to the hathing media. Since the vulnerability to NMDA receptor agonists in organotypic culture is correlated with the density of NMDA receptors in vivo, receptor distribution is probably the most important factor determining vulnerability in culture. Differences in glucose metabolism, rates of activity, or synaptic connectivity do not seem to be the primary determinants of vulnerability. The results in organotypic culture do not contradict the hypothesis of a ventricular concentration of the toxic endogenous agonist, since some differences remain in vivo. These differences may, however, arise from local differences within hippocampal regions that are not expressed in organotypic culture, and proximity to the ventricle may be coincidental.

In contrast, vulnerability to ischemic neuronal injury does not correlate well with either NMDA receptor distribution or vulnerability to direct exposure to NMDA receptor antagonists, even though in some models of ischemia (Simon et al., 1984; Gill et al., 1988; Choi, 1990; Vornov and Coyle, 1991a), including organotypic culture (Newell et al., 1990; Vornov et al., $1991 \mathrm{~b}$ ), injury can be prevented by NMDA receptor antagonists. The dentate gyrus is the hippocampal region most resistant to injury (Kirino, 1982), even though it possesses a high density of NMDA receptors and is quite sensitive to injury by hypoglycemia and NMDA. In ischemia, distribution of another receptor type or other neuronal characteristic must be the major determinant of regional injury.

Taken together, these observations suggest that the aspartate release during and after hypoglycemia results in direct, toxic activation of NMDA receptors in hippocampal areas with the highest density of NMDA receptors. The consistent protective effects of MK-801 administered $30 \mathrm{~min}$ after the end of 2-DG exposure suggest that aspartate's postsynaptic toxic effects are prolonged. A number of pre- or postsynaptic mechanisms could account for the prolonged protective effects of MK-801.

While it is possible that aspartate continues to accumulate in toxic concentrations for long periods after removal of the 2-DG, in vivo microdialysis studies have generally reported that extracellular accumulation of amino acids ends shortly after both hypoglycemia (Sandberg et al., 1986; Westerberg et al., 1988) and ischemia (Benveniste et al., 1984). In the present experiments, exchange with the large dilution volume of the culture medium would be expected to hasten the diffusion of agonist away from neurons, further shortening the period of accumulation of potential toxins. As noted above, the duration of block of glycolysis by 2-DG in organotypic culture has not yet been examined. An important first observation would be to confirm that tissue aspartate levels rise in organotypic culture and to correlate this rise with the time course of protection by MK801. Aspartate release may be prolonged, but it may not occur continuously at high concentrations. Alternatively, during re- covery, a different endogenous, selective NMDA receptor agonist could be released. Microdialysis experiments have demonstrated a moderate rise in quinolinic acid levels in the striatum during recovery from hypoglycemia (Westerberg et al., 1991). Since quinolinic acid is a selective agonist at the NMDA receptor, it could contribute to the NMDA receptor activation against which MK-801 protects. Extracellular aspartate or other agonist concentrations may not be elevated to levels that are normally toxic, but alterations in postsynaptic sensitivity to effects of endogenous agonists might be responsible for injury. Increased postsynaptic sensitivity to the toxic effects of endogenous agonists could result from the preceding pathologic events, that is, exposure of neurons to high concentrations of aspartate under conditions of relative substrate deprivation. For example, we have previously demonstrated that, in acute adult hippocampal slices, the sensitivity to NMDA receptor-mediated toxicity may be modified by ionic events (Vornov and Coyle, 1991a,b).

The time course of protection by TTX was not the same as that of MK-801. This provides evidence that the endogenous agonist responsible for injury is not released by normal synaptic transmission. TTX should block axonal conduction and physiologic neurotransmitter release, so that its presynaptic effects should be correlated with the postsynaptic effects of receptor antagonists. Since MK-801 protects long after TTX protects, we must assume that, after $30 \mathrm{~min}$ of recovery, the agonist release does not occur by the usual TTX-sensitive mechanisms. Nevertheless, the early protective effects of TTX suggest that sodium entry is necessary for prolonged aspartate release to occur. Large shifts in extracellular ion concentrations are well described in hypoglycemia (Astrup and Norberg, 1976; Harris et al., 1984) and are not dependent on NMDA receptor activation since they are not altered by systemic administration of MK-801 at doses that protect against neuronal degeneration (Zhang et al., 1990). This would be consistent with the idea that sodium entry is primary and can lead secondarily to toxic NMDA receptor activation. Sodium would accumulate intracellularly, entering through TTX-sensitive channels, and could secondarily result in an inward calcium flux through $\mathrm{Na}^{+} / \mathrm{Ca}^{2+}$ exchange, resulting in increased intracellular calcium and calcium-dependent neurotransmitter release. Additional aspartate release could also occur as sodium enters the intracellular space by causing the sodium-dependent glutamate transporter to run in reverse, transporting aspartate and sodium out of the cell, rather than using the normal sodium gradient for uptake of amino acids from the extracellular space. It should be noted that all of these mechanisms could occur in either glia or neurons.

It is also possible that block of sodium channels has metabolic effects, either preserving ATP levels or decreasing conversion of glutamate to aspartate. In vivo, ATP levels remain near normal in hypoglycemic animals until after an initial ionic transient (Harris et al., 1984; Wieloch et al., 1984), suggesting that early ionic shifts in hypoglycemia cause the metabolic stress in hypoglycemia that eventually results in ATP depletion and toxic NMDA receptor activation. Using the more accessible tissue culture model described here, these possibilities could be further evaluated with biochemical measurements. To our knowledge, while protective effects of TTX have been described in some animal models of ischemia (Prenen et al., 1988; Yamasaki et al., 1991), the cffects of TTX have not becn cxamined previously in hypoglycemia.

The experiments reported here describe a number of phenomena that suggest that much of the pathophysiology of hy- 
poglycemic hippocampal injury can be reproduced in organotypic culture. The distribution of injury seems to be a consequence of the regional vulnerability to the direct toxic activation of NMDA receptors by aspartate, which is simply determined by NMDA receptor density. The distribution, time course, and mechanism of aspartate release remain unexplored. Organotypic culture provides the first tissue culture model in which TTX is protective and in which delayed administration of MK-801 can be protective. Understanding of the injury mediated by prolonged, nonsynaptic release of endogenous neurotransmitters may be central to the development of practical strategies to protect the nervous system from injury during recovery from metabolic insults.

\section{References}

Agardh C-D, Folbergrova J, Siesjo BK (1978) Cerebral metabolic changes in profound insulin-induced hypoglycemia, and in the recovery period following glucose administration. J Neurochem 31: 1135-1142.

Astrup J, Norberg K (1976) Potassium activity in cerebral cortex in rats during severe insulin hypoglycemia. Brain Res 103:418-423.

Auer RN, Siesjo BK (1988) Biological differences between ischemia, hypoglycemia, and epilepsy. Ann Neurol 24:699-707.

Auer RN, Wieloch T, Olsson Y, Siesjo BK (1984) The distribution of hypoglycemic brain damage. Acta Neuropathol (Berl) 64:177-191.

Auer RN, Kalimo H, Olsson Y, Siesjo BK (1985a) The temporal evolution of hypoglycemic brain damage. II. Light- and electronmicroscopic findings in the hippocampal gyrus and subiculum of the rat. Acta Neuropathol (Berl) 67:25-36.

Auer RN, Kalimo H, Olsson Y, Wieloch T (1985b) The dentate gyrus in hypoglycemia. Pathology implicating excitotoxin-mediated neuronal necrosis. Acta Neuropathol (Berl) 67:279-288.

Barry WH, Peeters GA, Rasmussen CAF, Cunningham MJ (1987) Role of changes in $\left[\mathrm{Ca}^{2+}\right]_{i}$ in energy deprivation contracture. Circ Res 61:726-734.

Benveniste H, Drejer J, Schousboe A, Diemer NH (1984) Elevation of the extracellular concentrations of glutamate and aspartate in rat hippocampus during transient cerebral ischemia monitored by intracerebral microdialysis. J Neurochem 43:1369-1374.

Choi DW (1990) Cerebral hypoxia: some new approaches and unanswered questions. J Neurosci 10:2493-2501.

Diemer NH, Ekstrom von Lubitz DK (1983) Cerebral ischaemia in the rat: increased permeability of post-synaptic membranes to horseradish peroxidase in the early post-ischaemic period. Neuropathol Appl Neurobiol 9:403-414.

Facci L, Leon A, Skaper SD (1990) Hypoglycemic neurotoxicity in vitro: involvement of excitatory amino acid receptors and attenuation by monosialoganglioside GM1. Neuroscience 37:709-716.

Favaron M, Manev H, Alho H, Bertolino M, Ferret B, Guidotti A, Costa E (1988) Gangliosides prevent glutamate and kainate neurotoxicity in primary neuronal cultures of neonatal rat cerebellum and cortex. Proc Natl Acad Sci USA 85:7351-7355.

Gähwiler BH (1981) Organotypic monolayer cultures of nervous tissue. J Neurosci Methods 4:329-342.

Gill R, Foster AC, Woodruff GNB (1988)MK-801 is neuroprotective in gerbils when administered during the post-ischaemic period. Neuroscience 25:847-855.

Greenamyre JT, Olson JMM, Penney JB, Young AB (1985) Autoradiographic characterization of $N$-methyl-D-aspartate-, quisqualate-, and kainate-sensitive glutamate binding sites. J Pharmacol Exp Ther 233:254-263.

Hagberg H, Lehmann A, Sandberg M, Nystrom B, Jacobson I, Hamberger A (1985) Ischemia-induced shift of inhibitory and excitatory amino acids from intra- to extracellular compartments. J Cereb Blood Flow Metab 5:413-419.

Harris RJ, Wieloch T, Symon L, Siesjo BK (1984) Cerebral extracellular calcium activity in severe hypoglycemia: relation to extracellular potassium activity and energy state. J Cereb Blood Flow Metab 4: 187-193.

Johansen FF, Jorgensen MB, Ekstrom von Lubitz DK, Diemer NH (1984) Selective dendrite damage in hippocampal CA1 stratum ra- diatum with unchanged axon ultrastructure and glutamate uptake after transient cerebral ischaemia in the rat. Brain Res 291:373-377.

Jones KH, Senft JA (1985) An improved method to determine cell viability by simultaneous staining with fluorescein diacetate-propidium iodide. J Histochem Cytochem 33:77-79.

Kirino T (1982) Delayed neuronal death in the gerbil hippocampus following ischemia. Brain Res 239:57-69.

Lemasters JJ, DiGuiseppi J, Neiminen A-L, Herman B (1987) Blebbing, free $\mathrm{Ca}^{2+}$ and mitochondrial membrane potential preceding cell death in hepatocytes. Nature 325:78-81.

Macklis JD, Madison RD (1990) Progressive incorporation of propidium iodide in cultured mouse neurons correlates with declining elcetrophysiological status: a fluorescence scale of membrane integrity. J Neurosci Methods 31:43-46.

Monaghan DT, Holets VR, Toy DW, Cotman CW (1983) Anatomical distributions of four pharmacologically distinct ${ }^{3} \mathrm{H}$-glutamate binding sites. Nature 306:176-179.

Monyer H, Choi DW (1988) Morphinans attenuate cortical neuronal injury induced by glucose deprivation in vitro. Brain Res 446:144148.

Monyer H, Goldberg MP, Choi DW (1989) Glucose deprivation neuronal injury in cortical culture. Brain Res 483:347-354.

Newell DW, Malouf AT, Franck JE (1990) Glutamate-mediated selective vulnerability to ischemia is present in organotypic cultures of hippocampus. Neurosci Lett 116:325-330.

Papagapiou MP, Auer RN (1990) Regional neuroprotective effects of the NMDA receptor antagonist MK-801 (dizocilpine) in hypoglycemic brain damage. J Cereb Blood Flow Metab 10:270-276.

Prenen GH, Go KG, Postema F, Zuiderveen F, Korf J (1988) Cerebral cation shifts in hypoxic-ischemic brain damage are prevented by the sodium channel blocker tetrodotoxin. Exp Neurol 99:118-132.

Sandberg M, Butcher SP, Hagberg H (1986) Extracellular overflow of neuroactive amino acids during severe insulin-induced hypoglycemia: in vivo dialysis of the rat hippocampus. J Neurochem 47:178-184.

Simon RP, Swan JH, Griffiths T, Meldrum BS (1984) Blockade of $N$ methyl-D-aspartate receptors may protect against ischemic damage in the brain. Science 226:850-852.

SimorRPSchmidley WMeldrunBSSwar HChapmanAG (1986)Excitotoxic mechanisms in hypoglycemic hippocampal injury. Neuropathol Appl Neurobiol 12:567-576.

Tasker RC, Vornov JJ, Coyle JT (1991) IIypoglycemic injury in organotypic hippocampal culture is similar to NMDA neurotoxicity and is prevented by delayed MK-801. Soc Neurosci Abstr 17:1271.

Vornov JJ, Coyle JT (1991a) Enhancement of glutamate toxicity in the hippocampal slice by depolarization and ischemia. Brain Res 555: 99-106.

Vornov JJ, Coyle JT (1991b)Glutamate neurotoxicity and the inhibition of protein synthesis in the hippocampal slice. J Neurochem 56:996-1006.

Vornov JJ, Tasker RC, Coyle JT (1991a) Direct observation of the agonist-specific regional vulnerability to glutamate, NMDA, and kainate neurotoxicity in organotypic hippocampal cultures. Exp Neurol 114:11-22.

Vornov JJ, Tasker RC, Coyle JT (1991b) Early neuronal membrane injury precedes very delayed histologic degeneration after "ischemia" in organotypic hippocampal culture. Soc Neurosci Abstr 17:1093.

Westerberg E, Kehr J, Ungerstedt U, Wieloch T (1988) The NMDAantagonist MK-80I reduces extracellular amino acid levels during hypoglycemia and prevents striatal damage. Neurosci Res Commun 3:151-158.

Westerberg E, Magnusson K, Wieloch T, Ungerstedt U, Speciale C, Schwarcz R (1990) Extracellular levels of quinolinic acid are moderately increased in rat neostriatum following severe insulin-induced hypoglycaemia. Acta Physiol Scand 138:417-422.

Wieloch T (1985) Hypoglycemia-induced neuronal damage prevented by an $N$-methyl-D-aspartate antagonist. Science 230:681-683.

Wieloch T, Harris RJ, Symon L, Siesjo BK (1984) Influence of severe hypoglycemia on brain extracellular calcium and potassium activities, energy and phospholipid metabolism. J Neurochem 43:160-168.

Yamasaki Y, Kogure K, Hara H, Ban H, Akaike N (1991) The possible involvement of tetrodotoxin-sensitive ion channels in ischemic neuronal damage in the rat hippocampus. Neurosci Lett 121:251-254.

Zhang E, Hansen AJ, Wieloch T, Lauritzen M (1990) Influence of MK- 801 on brain extracellular calcium and potassium activities in severe hypoglycemia. J Cereb Blood Flow Metab 10:136-139. 